Sitzungsberichte

der Akademie der Wissenschaften der DDR

Mathematik - Naturwissenschaften - Technik

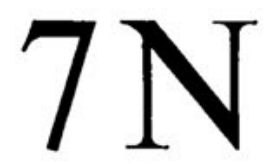

1984

\title{
Irmgard Garz
}

\section{Korrosionsverhalten} plastisch verformter Metalle 

Sitzungsberichte

der Akademie der Wissenschaften

der DDR

Jahrgang $1984 \cdot \mathrm{Nr} .7 / \mathrm{N}$

Mathematik - Naturwissenschaften - Technik

Irmgard Garz

Korrosionsverhalten

plastisch verformter Metalle

AKADEMIE-VERLAG $\cdot$ BERLIN

1984 
Vortrag von Frau Prof. Dr. sc. Irmgard Garz, Technische Hochschule „Otto von Guericke“ Magdeburg, gehalten am 8.12. 1983 vor der Klasse Werkstoffwissenschaft der Akademie der Wissenschaften der DDR

Herausgegeben im Auftrage des Präsidenten der Akademie der Wissenschaften der DDR von Vizepräsident Prof. Dr. Heinrich Scheel

ISSN $0138-3965$

Erschienen im Akademie-Verlag, DDR-1086 Berlin, Leipziger Str. 3-4 (C) Akademie-Verlag Berlin 1984 Lizenznummer: $202 \cdot 100 / 72 / 84$

Printed in the German Democratic Republic Gesamtherstellung: VEB Druckhaus Köthen LSV 3015

Bestellnummer : 7634457 (2010/84/7/N)

00350 